\title{
Spin injection into a short DNA chain
}

\author{
X. F. Wang and Tapash Chakraborty \\ Department of Physics and Astronomy, The University of Manitoba, Winnipeg, Canada, R3T 2N2
}

\begin{abstract}
Quantum spin transport through a short DNA chain connected to ferromagnetic electrodes has been investigated by the transfer matrix method. We describe the system by a tight-binding model where the parameters are extracted from the experimental data and realistic metal energy bands. For ferromagnetic iron electrodes, the magnetoresistance of a 30-basepair Poly (G)-Poly(C) DNA is found to be lower than $10 \%$ at a bias of $<4 \mathrm{~V}$, but can reach up to $20 \%$ at a bias of $5 \mathrm{~V}$. In the presence of the spin-flip mechanism, the magnetoresistance is significantly enhanced when the spin-flip coupling is weak but as the coupling becomes stronger the decreasing magnetoresistance develops an oscillatory behavior.
\end{abstract}

PACS numbers: 87.14.Gg,72.20.Ee,72.25.-b

In recent years, a remarkable progress in direct measurements of electron transport through DNA has generated intense interest in DNA electronics 1, 2, 3, 4, 5, 6, 7, 8, 9, 10, 11, 12, 13. DNA is found to have diverse electronic properties depending on its structure and the environment around it [6]. The clear semiconductor behavior observed in a short DNA chain of 30basepair Poly $(\mathrm{G})$-Poly $(\mathrm{C})$ has been explained by a tightbinding model [2, 9, 11]. On the other hand, spin transport through nanostructures has, of late, been receiving considerable attention because of the possiblity of developing spin-based electronic devices [14]. Inspired by the broad interest in spin-injection into mesoscopic systems
15], we have investigated the quantum spin transport 10] through a short DNA chain connected to ferromagnetic electrodes. We predict an enhancement and oscillation of magnetoresistance in this system taking into account the realistic band structure of ferromagnetic Fe electrode and a spin-flip mechanism.

We consider a $p$-type semiconductor DNA chain of $N$ basepairs connected to a circuit via metal electrodes. The tight-binding Hamiltonian of the system is

$$
H=H_{d}+H_{L}+H_{R}+H_{d m}+H_{d p s}+H_{s p}
$$

where

$$
\begin{aligned}
H_{d} & =-\sum_{\sigma, n=1}^{N} \varepsilon_{d}^{\sigma} C_{n, \sigma}^{\dagger} C_{n, \sigma}-\sum_{\sigma, n=1}^{N-1} t_{d}^{\sigma}\left(C_{n, \sigma}^{\dagger} C_{n+1, \sigma}+C_{n+1, \sigma}^{\dagger} C_{n, \sigma}\right), \\
H_{L} & =-\sum_{\sigma, n \leq 0} \varepsilon_{m L}^{\sigma} C_{n, \sigma}^{\dagger} C_{n, \sigma}-\sum_{\sigma, n \leq 0} t_{m L}^{\sigma}\left(C_{n, \sigma}^{\dagger} C_{n+1, \sigma}+C_{n+1, \sigma}^{\dagger} C_{n, \sigma}\right), \\
H_{R} & =-\sum_{\sigma, n \geq N+1} \varepsilon_{m R}^{\sigma} C_{n, \sigma}^{\dagger} C_{n, \sigma}-\sum_{\sigma, n \geq N+1} t_{m R}^{\sigma}\left(C_{n, \sigma}^{\dagger} C_{n+1, \sigma}+C_{n+1, \sigma}^{\dagger} C_{n, \sigma}\right), \\
H_{d m} & =-\sum_{\sigma} t_{d m}\left(C_{0, \sigma}^{\dagger} C_{1, \sigma}+C_{1, \sigma}^{\dagger} C_{0, \sigma}\right)-\sum_{\sigma} t_{d m}\left(C_{N, \sigma}^{\dagger} C_{N+1, \sigma}+C_{N+1, \sigma}^{\dagger} C_{N, \sigma}\right), \\
H_{d p s} & =-\sum_{\sigma, n=1}^{N} \Sigma_{n}^{\sigma}(E) C_{n, \sigma}^{\dagger} C_{n, \sigma}, \\
H_{s p} & =-\sum_{\sigma, n=1}^{N} t_{d}^{s o} C_{n, \sigma}^{\dagger} C_{n, \bar{\sigma}}-\sum_{\sigma, n=1}^{N-1} t_{d}^{s}\left(C_{n, \sigma}^{\dagger} C_{n+1, \bar{\sigma}}+C_{n+1, \bar{\sigma}}^{\dagger} C_{n, \sigma}\right) .
\end{aligned}
$$

Here $C_{n, \sigma}^{\dagger}$ is the creation operator of electron with spin $\sigma$ on site $n(=1, \cdots, N)$ of the DNA chain, the left electrode $(n \leq 0)$, and the right electrode $(n \geq N+1)$. $H_{d}$ describes electrons (holes) of spin $\sigma$ in the DNA chain with the on-site energies $-\varepsilon_{d}^{\sigma}\left(\varepsilon_{d}^{\sigma}\right)$, which is equal to the highest occupied molecular orbit (HOMO) energy of each base-pair, and the hopping parameters $t_{d}^{\sigma}$ between neighboring sites. The HOMO energy band is then determined by $\varepsilon_{d}^{\sigma}$ and $t_{d}^{\sigma}$. The Fermi energy $E_{F}$ in the $p$-type DNA chain locates between the HOMO and lowest unoccupied molecular orbit (LUMO) bands and is closer to the HOMO band edge. Experimental results have indicated 


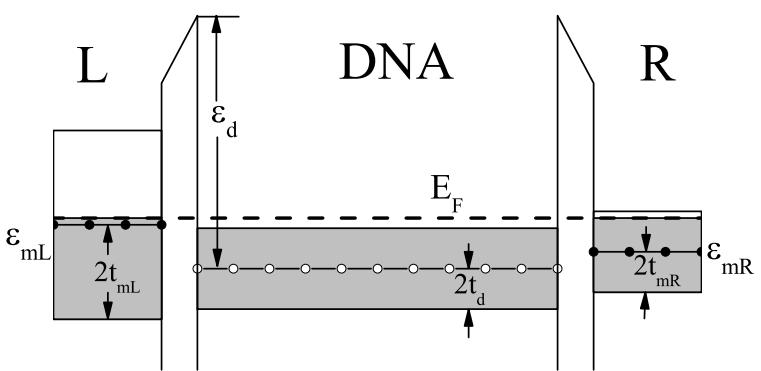

FIG. 1: Energy band of the system in equilibrium.

that the Fermi energy may vary from sample to sample 2]. $H_{X}$ with $X=L$ or $R$ denotes the Hamiltonian of electrons in the left electrode $(L)$ or the right electrode $(R)$. In the tight-binding model, $\varepsilon_{m X}^{\sigma}$ is the center of the energy band where the electrons are in the metals and $4 t_{m X}^{\sigma}$ its band width. When the DNA chain contacts to the metal electrodes, exchange of electrons (holes) between the DNA chain and the electrodes becomes possible. In equilibrium, as illustrated in Fig. 1 the Fermi energies of the electrodes and of the DNA match and a tunnelling barrier forms between them. The contact property is described by the tunnelling parameter $t_{d m}$. When a bias voltage drop is applied over the electrodes, distribution of the voltage drop or the potential profile along the non-equilibrium system depends on the DNA chain property and its contact with the metal electrodes. Since the free electron density in the metals is much higher than that in DNA, we assume that the band structure of the metal electrode is not changed by the applied bias. When the contact between DNA and the electrodes is poor, the voltage drop concentrates on the contact. In case of a perfect contact however, the whole voltage drop should be applied mainly along the DNA chain. In this letter, we assume the voltage drop is on the contact since it is supported by the fit to the experimental result (see below).

In real world, a DNA chain is composed of two strands of bases with one phosphate-sugar backbone connected to each strand. The backbones can affect the on-site energy of electrons in the basepairs. Further, the envi- ronment around the DNA chain may also play a role in the property of the electrons. Here we use a reservoir of semi-infinite chain [9, 16, 17] with a energy band of width $4 \gamma$ and a coupling of strength $\eta$ to each DNA basepair site to mimic the effect of the backbones and the environment. As a result, the on-site energy of each site in the DNA basepair is modified by a self energy $\Sigma_{n}^{\sigma}(E)$ which is energy dependent and is expressed as,

$$
\Sigma_{n}^{\sigma}(E)=\frac{\eta^{2}}{E-\varepsilon_{r}-\Sigma_{r}}
$$

with $\varepsilon_{r}$ being the on-site energy of the semi-infinite reservoir chain which we assume to be equal to the DNA onsite energy and $\Sigma_{r}=\left(E-\varepsilon_{r}\right) / 2-i\left[\gamma^{2}-\left(E-\varepsilon_{r}\right)^{2} / 4\right]^{1 / 2}$ the self energy of any reservoir site which is obtained self-consistently. In what follows, we have used the values $\eta=0.1 \mathrm{eV}$ and $\gamma=5 \mathrm{eV} 9$. To study the spin relaxation for a possible spin injection, we introduce the term $H_{s p}$ in the Hamiltonian to take into account the spin flip on-site and between neighboring sites described by the parameters $t_{d}^{s o}$ and $t_{d}^{s}$ respectively. The spin-flip along the DNA can be a result of spin-orbit interaction, magnetic impurity in the backbone, or magnetic environment.

In order to evaluate the transport properties of the system, we have employed the transfer matrix method [4, 5]. For an open system, the secular equation of the system is expressed as a group of infinite number of equations of the form,

$$
\begin{aligned}
t_{n-1, n} \Phi_{n-1}^{\sigma} & +t_{n-1, n}^{s} \Phi_{n-1}^{\bar{\sigma}}+\left(\varepsilon_{n}^{\sigma}+\Sigma_{n}^{\sigma}-E\right) \Phi_{n}^{\sigma}+t_{n}^{s o} \Phi_{n}^{\bar{\sigma}} \\
& +t_{n, n+1} \Phi_{n+1}^{\sigma}+t_{n, n+1}^{s} \Phi_{n+1}^{\bar{\sigma}}=0 .
\end{aligned}
$$

The wave functions of sites $n+1$ and $n$ are related to those of sites $n$ and $n-1$ by a transfer matrix $\hat{M}$,

$$
\left(\begin{array}{c}
\Phi_{n+1}^{+} \\
\Phi_{n+1}^{-} \\
\Phi_{n}^{+} \\
\Phi_{n}^{-}
\end{array}\right)=\hat{M}\left(\begin{array}{c}
\Phi_{n}^{+} \\
\Phi_{n}^{-} \\
\Phi_{n-1}^{+} \\
\Phi_{n-1}^{-}
\end{array}\right),
$$

with

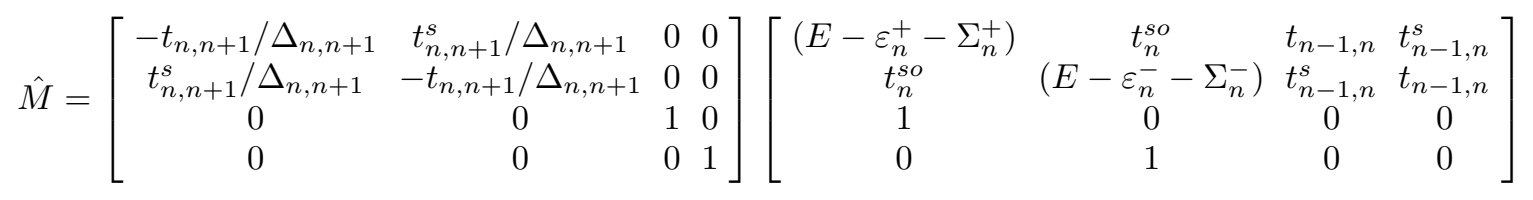

and $\Delta_{n, n+1}=\left(t_{n, n+1}\right)^{2}-\left(t_{n, n+1}^{s}\right)^{2}$. Assuming plane wave functions for the electrons $\Phi_{n}=\sum_{\sigma}\left(A^{\sigma} e^{i k_{L} n a}+\right.$
$\left.B^{\sigma} e^{-i k_{L} n a}\right)$ for $n \leq 0$ and $\Phi_{n}=\sum_{\sigma} C^{\sigma} e^{i k_{L} n a}$ for $n \geq$ $N+1$ in the left and right electrodes respectively, we can express the output wave amplitude $C^{\sigma}$ in terms of the 


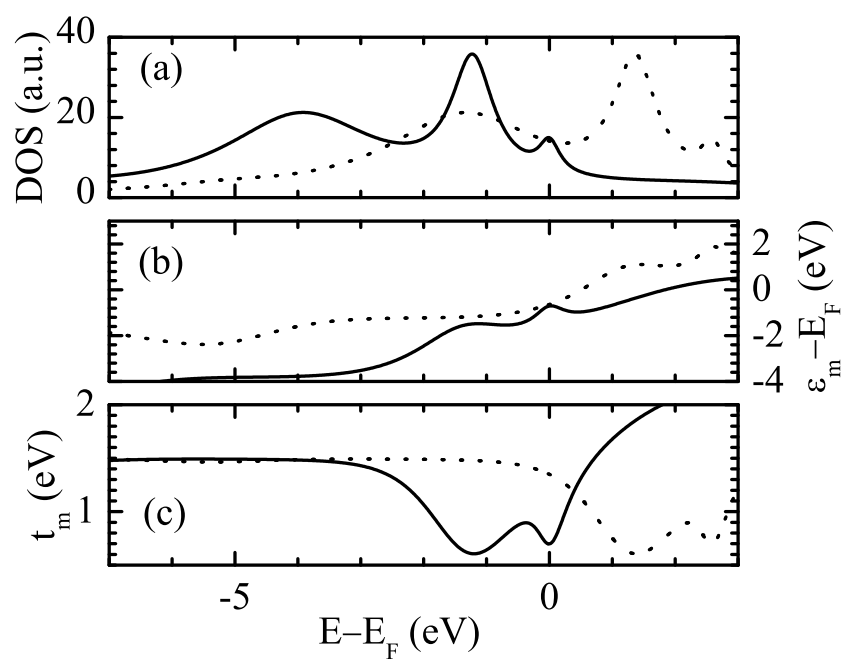

FIG. 2: Energy dependence of the parameters $\varepsilon_{m}$ (b) and $t_{m}$ (c) for ferromagnetic Fe. Solid curves and dotted curves correspond to spin-up and spin-down electrons. The resulting bulk DOS is also shown in (a).

input wave amplitude $A^{\sigma}$ and the transmission,

$$
T^{ \pm}(E)=\frac{\left|C^{ \pm}\right|^{2} \sin \left(k_{R}^{ \pm} a\right)}{\left|A^{+}\right|^{2} \sin \left(k_{L}^{+} a\right)+\left|A^{-}\right|^{2} \sin \left(k_{L}^{-} a\right)} .
$$

We choose a normalized incident amplitude $A^{ \pm}=$ $1 / \sqrt{\left|\sin \left(k_{L}^{ \pm} a\right)\right|}$. The net current primarily comes from transmission of electrons of energy between the electrodes' Fermi energies and is calculated as [17]

$$
I=\frac{e^{2}}{h} \sum_{\sigma} \int_{-\infty}^{\infty} d E T^{\sigma}(E)\left[f_{L}(E)-f_{R}(E)\right]
$$

with the Fermi function $f(E)=1 / \exp \left[\left(E-E_{F}\right) / k_{B} T\right]$ and the room temperature $T=300 \mathrm{~K}$. For ferromagnetic electrodes the megnetoresistance is defined as the percentage change of resistance between parallel and antiparallel configurations $R_{m}=\left(R_{\text {anti }}-R_{\text {paral }}\right) / R_{\text {anti }}=$ $\left(I_{\mathrm{p} a r a l}-I_{\mathrm{anti}}\right) / I_{\mathrm{p} a r a l}$.

In metals, an electron of energy $E$ may come from different energy bands. The corresponding effective parameters $\varepsilon_{m X}^{\sigma}$ and $t_{m X}^{\sigma}$ are then an average of the parameters of these bands and are energy dependent. In linear or quasi-equilibrium system, they are approximately the values near the Fermi energy. In a non-equilibrium system, if the difference of the Fermi levels between the two electrodes is comparable to the energy band width of the metals, the energy dependence should be taken into account. In the existing experiments the applied bias can be higher than $4 \mathrm{eV}$, which is larger than the width of the $d$ bands where the Fermi level locates in many metals. In the case of Ferromagnetic Fe which exemplifies the electrode material here, approximately five bands can be identified from the density of states (DOS)
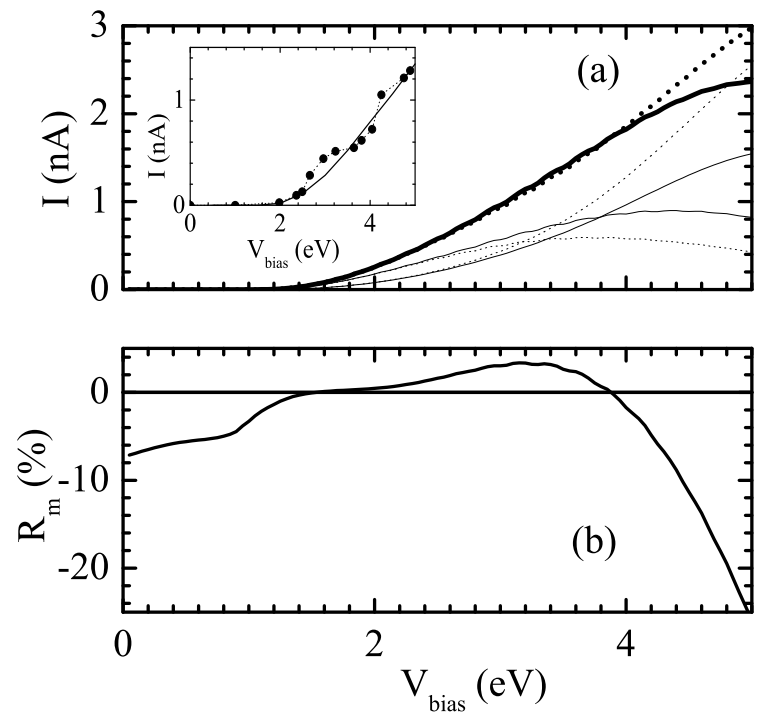

FIG. 3: (a) The I-V curve of a 30-basepair Poly (G)-Poly (C) DNA between two ferromagnetic Fe electrodes of parallel (solid curves) and anti-parallel (dotted) configurations. The thicker curves illustrate the total currents and the thinner ones the contribution from the two spin branches. (b) The magnetoresistance vesus the applied bias potential. In the inset of (a), we show our theoretical fit (thick solid curve) to the experimental result (filled circles connected by dotted lines) in Ref. 2].

near the Fermi energy of the bulk material [18]. For the spin-up (majority) electrons, the five bands locate approximately at $2.5,0,-0.68,-3.4$, and $-7 \mathrm{eV}$ above the Fermi energy with band width $6,0.3,0.6,4.1$, and $3.7 \mathrm{eV}$ respectively. For the spin-down (minority) electrons, the energy bands are the same as above but shifted $2.58 \mathrm{eV}$ to higher energy. Using Lorentzian broadening, we can mimic the bulk DOS and extract the parameters $\varepsilon_{m}^{\sigma}$ and $t_{m}^{\sigma}$ as shown in Fig. 2] At the Fermi energy, we get the hopping parameters $0.39 \mathrm{eV}$ and $1.62 \mathrm{eV}$ for spin-up and spin-down electrons respectively which coincide with the result obtained from the Fermi velocity [10].

Just as in Ref. [9], we extract the parameters of the DNA chain by fitting the experimental result of Ref. 2]. By evaluating the energy-dependent parameters $\varepsilon_{m}^{\sigma}$ and $t_{m}^{\sigma}$ from Platinum's band structure [19], we can fit the experimental result as shown in the inset of Fig. 3(a). As a result, we find that the hopping parameter is $t_{d}=0.6$ $\mathrm{eV}$, the equilibrium Fermi energy is $1.73 \mathrm{eV}$ higher than the DNA HOMO on-site energy, the contact parameters are $t_{d m}=0.019 \mathrm{eV}$ for the right electrode and $0.013 \mathrm{eV}$ for the left, $1 / 3$ of the bias voltage drop at the right contact and $2 / 3$ at the left. The above parameters are close to those obtained in Ref. [9] except for a larger $t_{d m}$ in the present case.

For ferromagnetic Fe electrodes as shown in Fig. 3(a), we get two I-V curves corresponding to the parallel (solid curves) and antiparallel (dotted curves) magnetization 


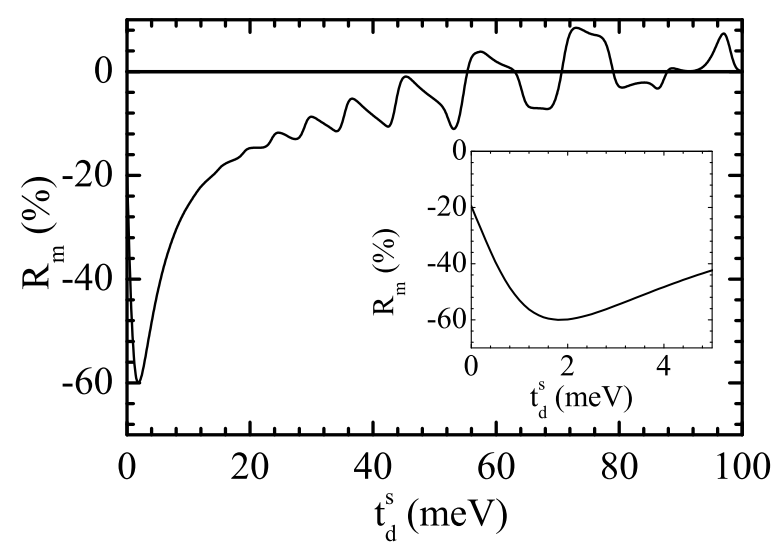

FIG. 4: The magnetoresistance vesus the spin-flip parameter $t_{d}^{s}$ in DNA when a bias of $4.8 \mathrm{eV}$ is applied. The other parameters are the same as in Fig. 3

configurations for the left and right electrodes, using the same contact parameter $t_{d m}=0.02 \mathrm{eV}$ for the two electrodes. In Fig. 3 (b), the magnetoresistance which describe the percentage change of the resistance of the system when being switched from parallel to antiparallel configuration, is plotted. In contrast to the results where constant values of the parameters $\varepsilon_{m}^{\sigma}$ and $t_{m}^{\sigma}$ at the Fermi energy were used, here we find a much smaller magnetoresistance until a strong bias is applied. This can be understood from the energy dependence of $t_{m}$ shown in Fig. 2(c). Instead of two parallel lines at $1.4 \mathrm{eV}$ and 0.6 $\mathrm{eV}$, the curves of $t_{m}$ vs $E$ for spin-up and spin-down electrons cross near the Fermi energy. This crossing makes the magnetoresistance disappear around the bias volt- age $V_{\text {bias }}=2 \mathrm{eV}$. The increase of magnetoresistance at higher bias voltage results from the increasing $t_{m}$ spread between spin-up and spin-down electrons in the range of $2 \mathrm{eV}$ around the Fermi energy.

In Fig. 4] we show how a spin-flip mechanism can affect the spin injection, assuming that the spin-flip can happen only when an electron jump from one site to another. The magnetoresistance does not decay monotonically to zero. Instead, the magnetoresistance is enhanced when there is a very weak spin-flip coupling $\left(t_{d}^{s}<1.9 \mathrm{meV}\right)$ as a result of the quantum interference in the system. In the transmission spectrum, peaks are slightly split with the increase of $t_{d}^{s}$, indicating the mixing of the spin-up and spin-down states in the system due to the spin-flip coupling. We observe an increase in magnetoresistance from $20 \%$ at $t_{d}^{s}=0$ to $60 \%$ at $t_{d}^{s}=1.9 \mathrm{meV}$ as displayed in the inset of Fig. [4 Then the magnetoresistance decreases smoothly until $t_{d}^{s}=20 \mathrm{meV}$. Above that value, the magnetoresitance begins to oscillate when it decays to zero.

In summary, we have investigated the quantum spin transport through a short DNA chain connected to ferromagnetic electrodes. We have used a tight-binding model to describe the system where the parameters are extracted from the experimental results and realistic energy bands of metals. We find that the energy band structure of the ferromagnetic electrodes significantly affects the resulting spin transport. In the presence of the spin-flip mechanism, enhancement and oscillation of magnetoresistance due to mixing of spin states are also observed.

The work of T.C. has been supported by the Canada Research Chair Program and the Canadian Foundation for Innovation (CFI) Grant.
[†] Electronic mail: tapash@physics.umanitoba.ca

[1] E. Braun and K. Keren, Adv. Phys. 53, 441 (2004); C. Dekker and M.A. Ratner, Phys. World 14, 29 (2001).

[2] D. Porath, A. Bezryadin, S. de Vries, and C. Dekker, Nature, 403, 635 (2000).

[3] K.-H. Yoo, D.H. Ha, J.-O. Lee, J.W. Park, J. Kim, J.J. Kim, H.-Y. Lee, T. Kawai, and H.Y. Choi, Phys. Rev. Lett. 87, 198102 (2001).

[4] P. Carpena, P. Bernaola-galvan, P. Ch. Ivanov, and H.E. Stanley, Nature, 418, 955 (2002).

[5] S. Roche and E. Macia, Mod. Phys. Lett. B 18, 847 (2004); S. Roche, Phys. Rev. Lett. 91, 108101 (2003).

[6] D. Porath, G. Cuniberti, and R. Di Felice, Top Curr. Chem. 237, 183 (2004).

[7] R.G. Endres, D.L. Cox, and R.R. Singh, Rev. Mod. Phys. 76, 195 (2004).

[8] M. Hjort and S. Stafstrom, Phys. Rev. Lett. 87, 228101 (2001).

[9] Xin-Qi Li and Yijing Yan, Appl. Phys. Lett. 79, 2190 (2001).

[10] M. Zwolak and M. Di Ventra, Appl. Phys. Lett. 81, 925 (2002).
[11] G. Cuniberti, L. Graco, D. Porath, and C. Dekker, Phys. Rev. B 65, 241314 (2002).

[12] V.M. Apalkov and T. Chakraborty, Phys. Rev. B 71, 33102 (2005).

[13] J.A. Berashevich, A.B. Filonov, V.E. Borisenko, (unpublished).

[14] D.D. Awschalom, D. Loss, and N. Samarth (Eds.), Semiconductor Spintronics and Quantum Computation (Springer, 2002); Proceedings of the First International Conference on the Physics and Applications of Spin Related Phenomena in Semiconductors, edited by H. Ohno [Physica E 10 (2001)].

[15] C.-M. Hu and T. Matsuyama, Phys. Rev. Lett. 86, 66803 (2001).

[16] J.L. D'Amato and H.M. Pastawski, Phys. Rev. B 41, 7411 (1990).

[17] S. Datta, Quantum Transport (Cambridge University Press, 2005).

[18] M. Singh, C. S. Wang, and J. Callaway, Phys. Rev. B 11, 287 (1975).

[19] D.L. Rogers, Phys. Stat. Sol. (B) 66, K53 (1974). 